\title{
Uncommon Diagnosis of an Emphysematous Cystitis: a Case Report
}

\author{
Dana Seifert ${ }^{1}$, Suad Jaganjac ${ }^{1}$
}

\begin{abstract}
A case of a 79-year-old woman who developed emphysematous cystitis (EC) prior to scheduled lower back surgery, suffering from severe back pain was presented. She had neither of the known EC risk factors (diabetes mellitus, immunodeficiency, neurogenic bladder and recurrent urinary tract infections) and presented herself without classical signs of EC (dysuria, haematuria, abdominal pain, pollakiuria, pneumaturia). She had a persistent back pain that was masked by her chronic back condition, leukocytosis and increased C-reactive protein (CRP) concentrations. The abdominal ultrasound showed a suspiciously impeded, cloudy vision in the lower pelvis and a blurry, thickened presentation of the wall of the urinary bladder with high echogenicity. A CT scan of the abdomen was performed and confirmed the diagnosis of an emphysematous cystitis, as well as a secondary pyelonephritis Escherichia coli was isolated from urine and blood and successfully treated with ciprofloxacin. Contrast-enhanced CT imaging is the diagnostic method of choice.
\end{abstract}

Key words: emphysematous cystitis; computerised tomography; diagnosis; pyelonephritis; Escherichia coli; ciprofloxacin.
(1) Department of Radiology, Schoen Klinik Hamburg Eilbek, Germany

Correspondence:

SUAD JAGANJAC

E: suad_jaganjac@yahoo.de

\section{ARTICLE INFO}

Received: 27 November 2019

Revision received: 7 December 2019 Accepted: 8 December 2019

\section{INTRODUCTION}

Emphysematous cystitis (EC) is a relatively uncommon disease of the lower urinary tract characterised by a diffuse inflammation of the urinary bladder wall with presence of intraluminal and/or intramural gas. EC can be caused by various pathogens, in most cases as a result of a bacterial infection. It is most commonly found in elderly women suffering from diabetes mellitus, which is the primary risk factor for EC. It is associated with a fairly high mortality due to the risk of the developing secondary pyelonephritis and/or urosepsis, and therefore requires early diagnosis and adequate treatment. ${ }^{1-3}$

The report presented here is on the case of a 79-year-old female who developed EC prior to lower back surgery. She was suffering from severe back pain, which made it difficult to distinguish the cause of the symptoms at first.

This case shows an unusual and unsuspected lower urinary tract infection that was diagnosed by abdominal ultrasound with subsequent confirmation on CT imaging.

\section{CASE HISTORY}

A 79-year old female was transferred to the hospital with heavy back pain for surgical treatment of a known lumbar spinal stenosis. Her medical history included hypertension, hypothyroidism and arteriosclerosis. She did not suffer from diabetes mellitus.

Routine presurgical blood tests revealed a rapid increase of the inflammation factors with an elevated white blood cell count up to $12.2 / \mathrm{nl}$ and an elevated CRP of $297 \mathrm{mg} / \mathrm{l}$ with an unclear focus. A chest $\mathrm{x}$-ray was inconspicuous. Urinalysis (test strip) was strongly positive for erythrocytes and haemoglobin and weakly positive for nitrite; 
it was negative for leucocytes. She did not present herself with fever, nor did she suffer from any urinary infection-related symptoms, such as dysuria, pollakiuria, macrohaematuria or pneumaturia. The abdominal ultrasound (Figure 1) showed a suspiciously impeded, cloudy vision in the lower pelvis (a) and a blurry, thickened presentation of the wall of the urinary bladder with high echogenicity (b).

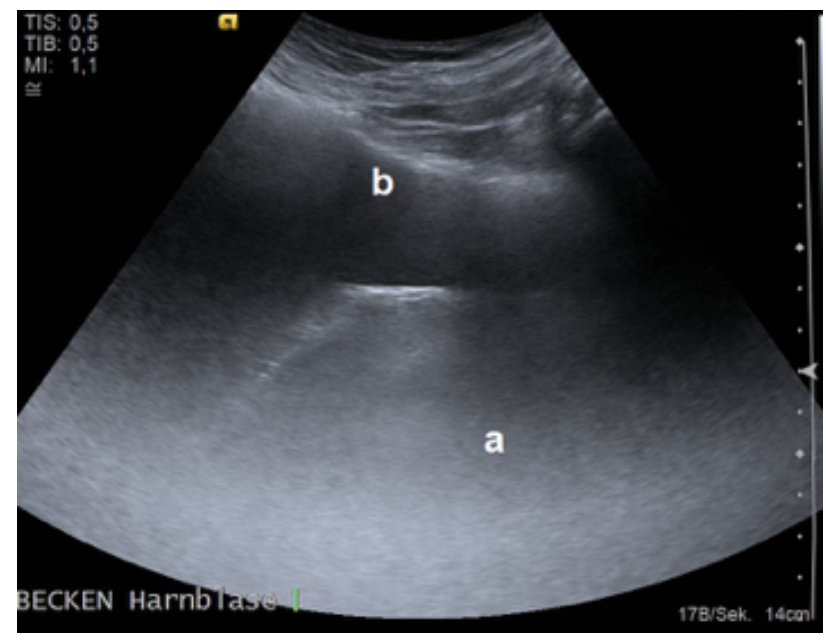

Figure 1: Ultrasound image of the pelvic region (a) including the urinary bladder (b)

The following CT scan of the abdomen (Fig. 2) confirmed the diagnosis of an emphysematous cystitis with countless gas bubbles both intramurally and intraluminally in the bladder, continuing into the wall of the right ureter (arrow), as well as a secondary pyelonephritis with ambilateral contrast uptake of the pelviocaliceal system (Fig. 3).

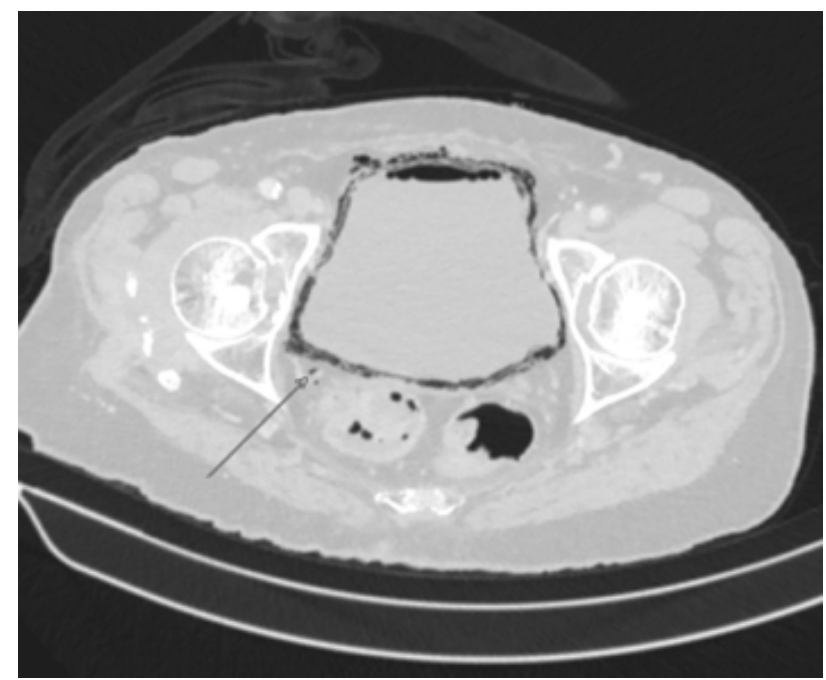

Figure 2: Contrast-enhanced CT of the abdomen (lung window) shows intramural and intraluminal air in the bladder, continuing within the wall of the right ureter (arrow)

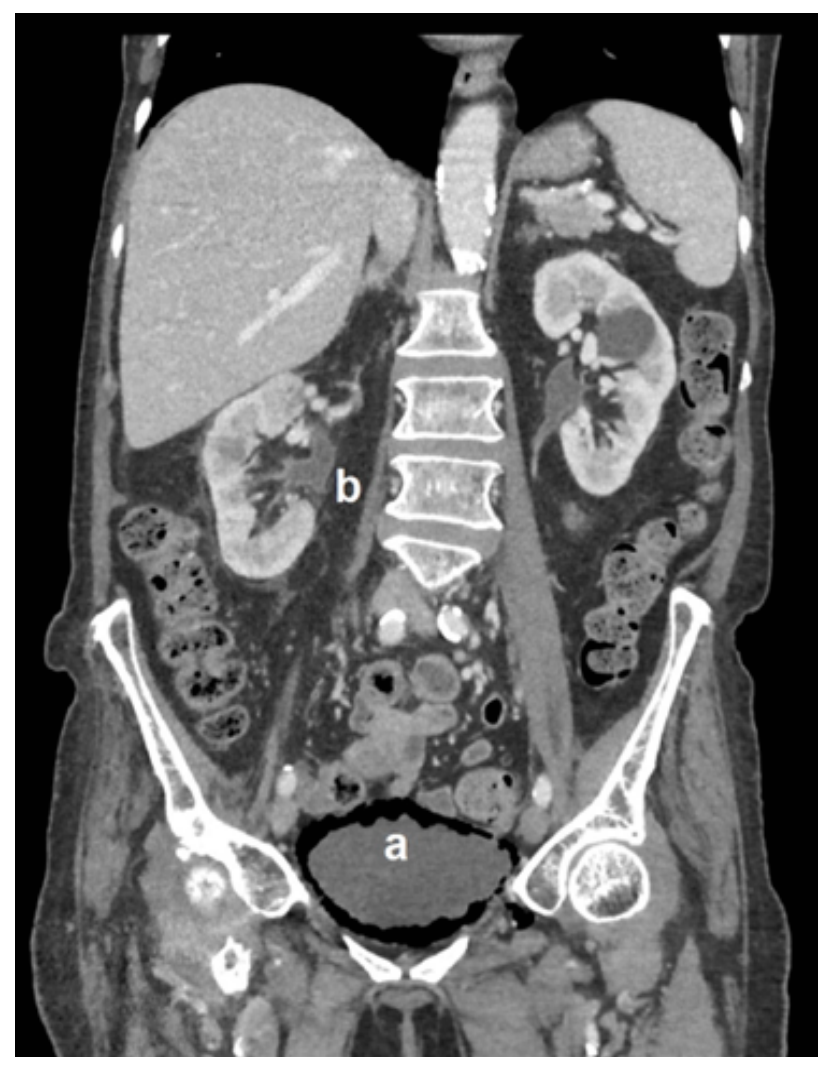

Figure 3: Coronal view of the contrast-enhanced abdominal CT shows intramural gas in the bladder (a), as well as an ambilateral contrast uptake of the pelviocaliceal system (b)

The patient was transferred to the intermediate care unit, where she was catheterised transurethrally for 8 days in total for sufficient bladder decompression and urinary drainage, and received intravenous antibiotics, initially ceftriaxone and metronidazole. Eventual microbial blood culture and culture of the urinalysis revealed Escherichia coli sensitive to ciprofloxacin, so the antibiotic therapy was altered accordingly.

The patient responded well to therapy with a quick decrease of inflammatory parameters and a good clinical recovery. She was dismissed from the intermediate care unit after four days and discharged from the hospital eight days after admission. The initially planned back surgery was postponed.

\section{DISCUSSION}

Emphysematous cystitis is a somewhat rare condition of the urinary bladder, mostly caused by bacterial infections. The most common organism causing EC is Escherichia coli (E. coli), followed by Klebsiella pneumoniae, Enterobacter 
and Clostridium species, Staphylococcus aureus, Proteus mirabilis, or Candida species. ${ }^{1,2}$ This corresponds well with the case presented here, as the patient's urinalysis and blood culture identified $E$. coli as the pathogen. EC is more common in elderly women in their 6 th and 7 th decade, with a 2:1 female vs male ratio. The main underlying conditions involve diabetes mellitus, immunodeficiency, neurogenic bladder and recurrent urinary tract infections., ${ }^{1,2,4}$ This patient did not suffer from any of the risk factors above, which is a special finding.

Symptoms are similar to those of uncomplicated cystitis and can range from dysuria, haematuria, abdominal pain, and pollakiuria, to fever and sepsis. A unique and typical symptom for EC is pneumaturia. ${ }^{1}$ This patient did not suffer from any of those listed symptoms, but a repeated back pain. In this case it was difficult to distinguish between the pre-existing back pain due to spinal stenosis and flank pain due to the later diagnosed secondary pyelonephritis.

Regarding imaging, ultrasound is usually the initial diagnostic investigation performed; it can show an abnormal diffuse urinary bladder wall thickening with high echogenicity along the wall and in the pelvis, such as in this patient. A plain radiography may also be helpful by revealing a radiolucent line of intramural gas bubbles outlining the bladder wall. The standard for confirming the diagnosis of EC and ruling out other pathologies such as enterovesical fistula is contrast-enhanced CT-imaging, as it is the most sensitive and specific diagnostic tool. ${ }^{1,3,5}$

Adequate therapy of EC includes sufficient bladder decompression and drainage via catheterisation. Antibiotic or antifungal coverage as per culture and sensitivity is also indispensable. ${ }^{1,6} \mathrm{In}$ this case, the identified pathogen was E. coli sensitive to ciprofloxacin. In case of diabetes mellitus, strict glyceamic control, as well as treatment of other relevant comorbidities are needed to secure a good outcome for the patient. In severe case of necrosis, bladder rupture, obstructions, anatomic abnormalities or secondary causes such as bladder stones, surgical intervention can also be necessary for treatment. ${ }^{1}$

Literature describes mortality rates of EC of up to $10 \% . .^{7-9}$ As a severe and potentially fatal condition, awareness and early detection as well as prompt treatment are necessary for a good recovery and outcome for the patient.

\section{CONCLUSION}

Emphysematous cystitis is an uncommon infectious disease of the urinary bladder with the detection of intramural and/or intraluminal gas. It has a strong female predominance and is mostly found in the elderly; main risk factors include diabetes mellitus and immunosuppression. Contrast-enhanced CT imaging is the diagnostic method of choice. Prompt recognition and adequate treatment, including sufficient drainage of the bladder, antibiotic therapy and strict glycaemic control, are required to prevent secondary complications such a urosepsis, and to secure a good outcome for the patient.

\section{ACKNOWLEDGEMENTS}

None.

\section{CONFLICT OF INTEREST}

None.

\section{REFERENCES}

1. Eken A, Alma E. Emphysematous cystitis: the role of CT imaging and appropriate treatment. Can Urol Assoc J 2013;7(11-12):E754-6.

2. Schicho A, Stroszczynski C, Wiggermann P. Emphysematous cystitis: mortality, risk factors, and pathogens of a rare disease. Clin pract 2017;7(2):930.doi: 10.4081/cp.2017.930. eCollection 2017 Apr 6.

3. Quint HJ, Drach GW, Rappaport WD, Hoffmann CJ. Emphysematous cystitis: areview of the spectrum of disease. J Urol 1992;147(1):134-7.

4. Ankel F, Wolfson AB, Stapczynski JS. Emphysematous cystitis: a complication of urinary tract infection occurring predominantly in diabetic women. Ann Emerg Med 1990;19(4):404-6.

5. Grupper M, Kravtsov A, Potasman I. Emphysematous cystitis: illustrative case report and review of the literature. Medicine 2007;86(1):47-53.

6. Sharma R, Mitra SK, Choudhary A, Majee P. Emphysematous cystitis-gas in bladder: a rare urological emergency. BMJ Case Rep 2015 Jul 23;2015. pii: bcr2015210836. doi: 10.1136/bcr-2015-210836.

7. Toyota N, Ogawa D, Ishii K, Hirata K, Wada J, Shikata K, et al. Emphysematous cystitis in a patient with type 2 diabetes mellitus. Acta Med Okayama 2011;65(2):129-33.

8. Thomas AA, Lane BR, Thomas AZ, Remer EM, Campbell SC, Shoskes DA. Emphysematous cystitis: A review of 135 cases. BJU Int 2007;100(1):17-20.

9. Kumar A, Turney JH, Brownjohn AM, McMahon MJ. Unusual bacterial infections of the urinary tract in diabetic patients--rare but frequently lethal. Nephrol Dial Transplant 2001;16(5):1062-5. 